\title{
Mapping Quantitative Trait Loci for Cold Tolerance in Rice under Germination Stage by Whole Genome Resequencing and Analysis of Candidate Genes
}

\section{Yinghua Pan}

Rice Research Institute, Guangxi Academy of Agricultural Sciences/Guangxi Key Laboratory of Rice Genetics and Breeding

\section{Lei Chen}

Rice Research Institute, Guangxi Academy of Agricultural Sciences/Guangxi Key Laboratory of Rice Genetics and Breeding

\section{Xinghai Yang}

Rice Research Institute, Guangxi Academy of Agricultural Sciences/Guangxi Key Laboratory of Rice Genetics and Breeding

\section{Lijun Gao}

Guangxi Academy of Agricultural Sciences/Guangxi Crop Genetic Improvement and Biotechnology Laboratory

\section{Baoxuan Nong}

Rice Research Institute, Guangxi Academy of Agricultural Sciences/Guangxi Key Laboratory of Rice Genetics and Breeding

\section{Xiuzhong Xia}

Rice Research Institute, Guangxi Academy of Agricultural Sciences/Guangxi Key Laboratory of Rice Genetics and Breeding

\section{Zongqiong Zhang}

Rice Research Institute, Guangxi Academy of Agricultural Sciences/Guangxi Key Laboratory of Rice Genetics and Breeding

\section{Chengcui Huang}

Rice Research Institute, Guangxi Academy of Agricultural Sciences/Guangxi Key Laboratory of Rice Genetics and Breeding

\section{Gaoxing Dai}

Rice Research Institute, Guangxi Academy of Agricultural Sciences/Guangxi Key Laboratory of Rice Genetics and Breeding

\section{Danting Li}

Rice Research Institute, Guangxi Academy of Agricultural Sciences/Guangxi Key Laboratory of Rice Genetics and Breeding 
Guangxi Academy of Agricultural Science https://orcid.org/0000-0003-0782-6158

\section{Original article}

Keywords: cold tolerance, germination, resequencing, quantitative trait locus, wild rice

Posted Date: November 5th, 2020

DOl: https://doi.org/10.21203/rs.3.rs-101299/v1

License: (c) (i) This work is licensed under a Creative Commons Attribution 4.0 International License. Read Full License 


\section{Abstract}

Low-temperature stress significantly affects rice growth and causes serious loss of yield in temperate and high-altitude areas of the world. Rice lacks cold tolerance (CT) at germination stage, which reduces seedling vigor, hinders crop establishment and crop growth, and even affect direct-seeded rice. A chromosome segment substitution line (CSSL) population, including 271 lines, was derived from coldtolerant donor wild rice Y11 (Oryza rufipogon Griff.) crossed with cold-sensitive rice variety GH998 to explore new genetic resource with CT and further investigate quantitative trait loci (QTLs) responsible for germination properties under low temperature. The germination rates of $\mathrm{Y} 11$ and $\mathrm{GH} 998$ were $95 \%$ and $73.33 \%$, respectively, under cold stress. The range of variance of the germination rate in the CSSLs was between $0 \%$ and $98.33 \%$. In addition, the CSSLs and parents were sequenced via whole genome resequencing. Results showed 33.46, 33.36, and $475.65 \mathrm{Gbp}$ of clean data of GH998, Y11, and CSSLs, respectively. In 12 linkage groups, the general map distance was $941.49 \mathrm{cM}$, while the average map distance was $0.63 \mathrm{cM}$. On thebasis of 1484 bins, a high-density linkage map of the CSSLs was developed. The average distance of the linkage map ranged from $0.42 \mathrm{cM}$ to $1.39 \mathrm{cM}$. The phenotype of CSSLs under low temperature and 615,466 single nucleotide polymorphisms (SNPs) between Y11 and GH998 were used for QTL analysis. Two QTLs, namely, low-temperature germination 8 ( $q$ LTG8) and qLTG11, were responsible for the germination ability under low temperature in rice. $q L T G 8$ was mapped on chromosome 8 , and it explained $14.55 \%$ of the total phenotypic variation explained (PVE) during the germination stage. $q L T G 8$ was in $195.5 \mathrm{~kb}$, and 32 genes were predicted based on the Rice Genome Annotation Project. qLTG11 was located on chromosome 11, and it explained $14.31 \%$ of the total PVE during the germination stage. qLTG11 was mapped at a narrow distance in $78.83 \mathrm{~kb}$, and only 12 genes fall within this range according to the Rice Genome Annotation Project. The expression patterns of these 32 genes in the qLTG8 region demonstrated that LOC_Os08g01120, LOC_Os08g01140, LOC_Os08g01390, LOC_Os08g01170, and LOC_Os08g01380 were highly induced by cold stress in Y11 compared with GH998. The expression patterns of 12 genes in the $q L T G 11$ region suggested that LOC_Os11g32880 and LOC_Os11g32940 were highly induced by cold stress in Y11 compared with GH998. This study provides an effective method, i.e., constructing CSSLs of wild rice, to explore excellent genes of wild rice and create new genetic resources. The results also provide a basis for identifying the genes underlying qLTG8 and $q L T G 11$, indicating that QTL could be used for genetic improvement of CT in cultivar rice.

\section{Introduction}

Extreme climate events make crops suffer from increasing abiotic stresses. Low temperature is one of the most common abiotic stresses that affects plant growth and reproduction, limits the geographical location of plant species, and reduce crop production (Ding et al. 2019). As rice originates from tropical and subtropical regions, it is appropriate to grow with optimal temperature $25^{\circ} \mathrm{C}-35^{\circ} \mathrm{C}$ (Xu et al. 2015). In high-latitude or high-altitude regions of Asia, Europe, America, and other rice cultivation areas, rice could not growth without a hitch because temperature is not consistently high. Rice growing in 25 countries suffered from low temperatures as a major climatic problem (Cruz et al. 2013). In China, rice is 
widely planted from Hainan island $\left(18^{\circ} 90^{\prime} \mathrm{N}\right)$ to the Mohe River $\left(53^{\circ} 27^{\prime} \mathrm{N}\right)$ in Heilongjiang. Cold injury leads to an estimated annual loss of approximately 3-5 million tons because rice production areas are subjected low temperature stress (Liu et al. 2019). Rice plants are more sensitive to cold stress during germination, seedling, tillering, panicle development, and booting stages (Zhao et al. 2017; Zhang et al. 2017). Chilling injury at the rice germination stage could lead to worsened germination rate, delayed seedling emergence, slowed growth, reduced rates of photosynthesis, and subsequently, fatal yield loss. For early direct-seeded rice, cold tolerance (CT) at the germination stage is an important characteristic (Yang et al. 2020). Therefore, exploring CT quantitative trait locus (QTL), selecting cold-resistant lines, cloning CT genes could provide insights into understanding the mechanisms and be beneficial to breeding chilling-tolerant cultivars for maintaining high and stable yields in rice cultivation regions.

CT is a quantitative trait controlled by multiple loci and influenced by the environment (Li et al. 2018; Zhang et al. 2017; Liu et al. 2019). In the last two decades, an increasingly number of QTLs mapped, isolated, and cloned has been noted. It helped elucidate the mechanisms of plant under cold stress. For example, six QTLs were related to CT at the germination stage on chromosomes $1,4,8$, and 11, as identified using backcross recombinant inbred lines (RILs) derived from a cross between indica cv. Changhui 891 and japonica cv. 02428 (Jiang et al. 2017). Two major QTLs qNGR1 and qNGR4 affected indica Changhui 891 germination (Jiang et al. 2017). Tomohiro detected four QTLs responsible for germination properties under low temperature on chromosomes 3 and 11 in an East European rice variety called Maratteli (Satoh et al. 2016). After exposure to cold $\left(14^{\circ} \mathrm{C}\right)$ for $7,11,14$, and $17 \mathrm{~d}$, the lowtemperature vigor of germination (LVG) and the cold response index for vigor of germination (CIVG) was used as evaluation indices; $q L V G 2$ was identified in RM29-RM262 on chromosome 2, qLVG7-2 and qCIVG7-2 were located in RM336-RM118 on chromosome 7, and qLVG7-1 and qCIVG7-1 explained up to $22.9 \%$ and $15.3 \%$ phenotypic variations, respectively (Han et al. 2006). Pan et al. evaluated the CT of 174 Chinese rice accessions at the germination and booting stages, and they found 51 QTLs by using genome-wide association studies (Pan et al. 2015). Yang et al. used RILs of highly-tolerant-to-lowtemperature indica rice $\mathrm{H} 335$ and sensitive-to-low-temperature indica rice CHA-1 to detect 11 QTLs on chromosome 9 on the basis of a high-density genetic map; six QTLs explained $5.13-9.42 \%$ of the total phenotypic variation during the germination stage (Yang et al. 2020). By using a RIL mapping population from a US weedy rice accession "PSRR-1," a rice cultivar "Bengal," and 212 simple sequence repeat markers, 49 QTLs distributed over 10 chromosomes were identified for 11 traits, along with three major QTLs associated with coleoptile length and seedling shoot length under low temperature (Borjas et al. 2016). Yang et al. mapped two cold-tolerant QTLs ( $q C T B B-5$ and $q C T B B-6)$ at the bud bursting in single segment substitution lines (SSSLs) derived from cold-tolerant japonica variety "Nan-yang-zhan"/indica variety "Huajing-xian 74"(Yang et al. 2016). Twelve QTLs for low-temperature germinability (LTG) were identified, and they could explain greater than $10 \%$ of the phenotypical variation (Yang et al. 2018). Comparison of low-temperature germination in the population (DX-BILs) for SLAF-seq showed that 94 BILs $\left(\mathrm{BC}_{1} \mathrm{~F}_{7}\right)$ derived from a hybrid between DXWR and Xieqingzao $\mathrm{B}$, five QTLs qLTG2, qLTG5, qLTG10.1, qLTG10.2, and $q L T G 12$ were separated, while $q L T G 5, q L T G 10.1$, and $q L T G 10.2$ could explain $19.7 \%$, $14.2 \%$, and $12.1 \%$ of the phenotypical variation, respectively (Li et al. 2019). Previous studies suggested 
that rice $\mathrm{CT}$ is a complex quantitative trait affected by the environment; it is highly complex and needs further in-depth exploration.

Few genes associated with cold tolerance have been cloned. Fujino et al. mapped and cloned a controlling low-temperature germinability gene $q L T G 3-1$ in rice (Fujino et al. 2008). qLTG3-1 was encoded by a protein of unknown function and strongly expressed in the embryo during seed germination (Fujino et al. 2008). Ma et al. used RILs generated from a cross between chilling-tolerant Nipponbare (japonica) and chilling-sensitive $93-11$ (indica) to fine mapped a QTL COLD1 associated with divergence in chilling tolerance (Ma et al. 2015). COLD1 interacted with $\mathrm{G}$ protein subunit RGA1 to sense low temperature (Ma et al. 2015). LTG5 was identified as a CT gene for low-temperature germinability (Pan et al. 2020). LTG1 is located on chromosome 2, and it encodes casein kinase and regulates the hypothermic response of rice. $L T G 1$ also influences auxin transport, synthesis, and signal transduction and regulates positively low temperature tolerance of rice during vegetative growth period (Lu et al. 2014). HAN1 is a major QTL located on chromosome 11, with an LOD value of 11.5 , which could explain $35.8 \%$ of the total phenotypic variation under low-temperature stress. HAN1 encodes an oxidase that catalyzes the conversion of biologically active jasmonoyl-L-isoleucine that regulated JA-mediated chilling response (Mao et al. 2019). qBSR10 showed CT in rice at the seedling stage (Xiao et al. 2018). The genes responsible for chilling tolerance were identified, revealing that genes affect seed germination. However, little is known about the wild rice gene with CT. In the present study, a high-density genetic map consisting of 1484 bin markers was obtained through 271 chromosome segment substitution lines (CSSLs) derived from wild rice Y11(Oryza rufipogon Griff.) and indica rice variety GH998. Two QTLs were explored from wild rice.

Here, a CSSL population was developed by CT donor wild rice Y11 (Oryza rufipogon Griff.) crossed with cold-sensitive rice variety GH998. In this study, evaluation of CT in parents and CSSL population and whole genome resequencing was performed to explore chilling tolerance-associated QTL for germination rate under severe chilling stresses at the germination stage. Through this approach, the QTLs for CT in Y11 were analyzed, and two QTLs were identified on chromosome 8, named qLTG8 (QTL for lowtemperature tolerance at germination stage on chromosome 8). Another one was located on chromosome 11, named qLTG11. The results contributed to the exploration of new QTLs for CT at the germination stage of wild rice Y11. The results also indicated that future cloning of the candidate gene could facilitate genetic bases for $\mathrm{CT}$ at the germination stage. Even the results could be applied in rice breeding program.

\section{Results}

\section{Sequencing and Genotyping of CSSLs and Their Parents}

In this study, 33.46 and $33.36 \mathrm{Gbp}$ clean data in GH998 and Y11 were obtained, respectively. The total data of $271 \mathrm{CSSLs}$ was $475.65 \mathrm{Gbp}$. The ratio of Q30 for each sample was above $85 \%$. A total of $1,561,817$ SNPs was detected between the parents, among which $1,181,012$ SNP markers, with the depth 
of not less than $4 X$, were used in CSSLs. A total of 1484 bin markers and 615,466 SNP markers were obtained to construct the recombination map. In the 12 linkage groups, the general map distance was $941.49 \mathrm{cM}$, and the average map distance was $0.63 \mathrm{cM}$. Resequencing generated 111,669,428, $111,350,954$ and 1,587,693,991 clean reads from GH998, Y11, and CSSLs, respectively (Table 1). The quality values of sequencing greater than or equal to 30 (Q30) percentages were above $91 \%$ of three libraries (Table 1). The percentage of mapped reads to reference genome in all clean reads in $\mathrm{GH} 998$ and Y11 were above $90 \%$ as properly mapped. The results showed that high quality of resequencing was achieved (Table 2). The sequencing depth and genome coverage of each sample were counted compared with those of reference genome. The SNP marker of chr2 was 116,138, the highest among all linkage groups (Table 3). The SNP marker of chr12 was 5055 less than that of other linkage groups (Table 3). Therefore, the SNP markers, bin, and linkage groups could construct genetic map effectively.

\section{Linkage Map of Recombination Bins}

A linkage map was developed using the 1,484 bins generated from whole-genome resequencing of the $271 \mathrm{CSSLS}$ and their parents. The average distance of the linkage map ranged from $0.42 \mathrm{cM}$ to $1.39 \mathrm{cM}$ (Table 4). The total max gap was the largest in the linkage group on chromosome 1 (15.55, Table 4). The max gap was the smallest was in the linkage group on chromosome 6 (3.96, Table 4). This result suggested that most genomes in CSSL contain a few chromosomes with no-recombination, indicating that they completely came from a single parent genome. Some chromosomes were heterozygous, which may be related to incomplete or faulty repair after chromosome exchange (Fig. 2a). Based on Bin information, Bin had been divided into 12 linkage groups. The Spearman coefficient between the linkage group and the map was near to 1 (Fig. 2b). The genetic map of $941.49 \mathrm{cM}$ that was constructed with linear arrangement of markers and genetic distance between adjacent markers (Fig. 2c). The single plant was checked for genotype. Most of the CSSLs have GH998 background, and Y11 fragments permeates in different chromosomes in the CSSL line (Supplementary Fig. 1). Linkage to all linkage groups of markers has been analyzed. The recombination rate between most markers was small (Supplementary Fig. 2).

\section{Phenotypic Evaluation of CSSLs and Their Parents}

In this study, one Guangxi common wild rice $\mathrm{Y} 11$ and one indica variety GH998 were crossed to develop the CSSL populations for QTL analysis and resequencing of CT. Overall, the parents and CSSL seeds were germinated at $28{ }^{\circ} \mathrm{C}$ after sowing, and the germinated seeds were treated under cold stress. Y11 showed $95 \%$ germination rate under cold stress compared with GH998 at $73.33 \%$ under LT (Table 5 and Fig. 3a). The germination rate under cold stress of the cold-tolerant parent $\mathrm{Y} 11$ was significantly higher than that of the cold-sensitive GH998 (Fig. 3a). The range of variance was between $0 \%$ and $98.33 \%$ (Table 5). The average germinate rate was $57.33 \%$, with a range of $0-98.33 \%$ under LT in the CSSL populations (Table 5 and Fig. $3 \mathrm{~b}$ ). These results suggested that $\mathrm{Y} 11$ is a CT wild rice compared with coldsensitive variety GH998. Among the CSSL populations, the absolute values of skewness and kurtosis were all near to 1 , indicating that the data were suitable for QTL analysis.

\section{QTL Analysis for germination rate under low temperature}


Based on sequence variations in Y11, GH998, and the CSSLs, 615,466 SNPs between Y11 and GH998 were used for QTL analysis in this study. The SNP between the recombination break point was classified as Bin, which was not a recombination event. A total of 1484 bins was analyzed using HighMap software to construct a genetic linkage map. The CT phenotype of the CSSL lines was checked for germination rate under $L T\left(6^{\circ} \mathrm{C}\right)$ for 8 days and recovered for 7 days (qLTG). The data of genetic linkage map and phenotype of CSSLs and their parents were used for QTL analysis. Two QTLs were identified for CT in the CSSL populations (Table 6). Two QTL peaks were detected on chromosomes 8 and 11. Then, two QTLs were mapped independently on rice chromosomes 8 and 11 (Fig. 4a). The QTL with CT on chromosomes 8 was named $q L T G 8$, while the other one was $q L T G 11$. The LOD values for $q L T G 8$ and $q L T G 11$ were 3.41 and 3.35, respectively. In addition, $q L T G 8$ and $q L T G 11$ explained $14.55 \%$ and $14.31 \%$ of the phenotypic variance, respectively (Table 6). Analyses showed that $q L T G 8$ was in a region of $66,986-262,445$ bp on chromosome 8 , while $q L T G 11$ was in a region of 19,424,787-19,503,621 bp on chromosome 11 (Figs. 4b and $\mathrm{c}$ ).

\section{Candidate Gene Analysis}

Resequencing analysis revealed that qLTG8 was located in a region of $195.5 \mathrm{~kb}$, and 32 genes were predicted based on the Rice Genome Annotation Project (http://rice.plantbiology.msu.edu/, Fig. 4b). Among those genes, five frequencies were for transcription. Three frequencies worked for replication, recombination, and repair. Three frequencies worked in signal transduction mechanisms. Two frequencies were involved in posttranslational modification, protein turnover, and chaperones. Two frequencies took part in energy production and conversion. Four frequencies were for general function prediction only (Fig. 5a). The transcripts of the candidate gene were divided in cellular component and molecular and biological processes. Five transcripts were found to be for response to stimulus. Three transcripts were involved in nucleic acid binding transcription factor activity. One transcript demonstrated a relation with transporter activity (Fig. 5c). QTL analysis showed that $q L T G 11$ was mapped in a narrow distance at $78.83 \mathrm{~kb}$, and only 12 genes fall within this range according to the Rice Genome Annotation Project (http://rice.plantbiology.msu.edu/, Fig. 4c). Among these genes, two frequencies were for transcription. Another two frequencies worked for replication, recombination, and repair. One frequency was for carbohydrate transport and metabolism. The transcripts of the candidate gene were divided in cellular component and molecular and biological processes. Four transcripts were for response to stimulus. Two transcripts exhibited a relation with catalytic activity(Fig. 5b, d). These results indicated that the functions of gene in the regions were analyzed.

\section{Candidate genes identified in the qLTG8 interval}

Thirty-two predictive genes (http://rice.plantbiology.msu.edu/) were found in the region of $195.5 \mathrm{~kb}$ on chromosome 8 (Supplementary Table S4). The expression levels of 32 genes in the bud were examined using quantitative real-time PCR (qRT-PCR). Then, 32 gene primers pairs for qRT-PCR were designed on the basis of cDNA sequences. Among them, 24 genes encoded known functional proteins, while three genes were annotated as encoding a protein kinase family protein, a zinc finger DHHC domain-containing protein, and a phosphatidylinositol-4-phosphate 5-Kinase protein. Four genes were annotated as 
encoding expressed proteins with unknown function; four were annotated as encoding expressed proteins with unknown function, one was annotated as encoding a hypothetical protein, two were annotated as encoding transposon proteins with unknown function, and two genes encoded unclassified retrotransposons (Table 6).

Among the 32 genes, the LOC_Os08g01120 in Y11 showed significantly higher expression levels than that in GH998. The expression of LOC_Os08g01140 in Y11 was also significantly higher than that in GH998 but decreased in $7 \mathrm{~d}$.

Three genes were induced by cold stress at different degrees between Y11 and GH998. LOC_Os08g01390 had significantly higher expression levels at all degrees in Y11 than in GH998 but not in $6 \mathrm{~d}$. The expression LOC_Os08g01170 in cold-tolerant Y11 was significantly higher than that in cold-sensitive GH998 at all degrees. LOC_Os08g01380 was induced during cold stress, and it had a high expression at $24 \mathrm{~h}, 3 \mathrm{~d}$, and $5 \mathrm{~d}$ in $\mathrm{Y} 11$, but a minimal difference between $\mathrm{Y} 11$ and GH998 was observed. These results suggested that LOC_Os08g01170 and LOC_Os08g01390 may be candidate genes, which encode an acetyltransferase protein, for the major QTL controlling CT in Y11 (Fig. 6a and Supplementary Fig. 3).

\section{Candidate genes identified in qLTG11 interval}

Twelve genes were predicted in $q L T G 11$ located at the $78.83 \mathrm{~kb}$ region according to the Rice Genome Annotation Project database (http://rice.plantbiology.msu.edu/). Among them, four genes encoded known functional proteins; two were annotated as encoding expressed proteins with unknown function; four were annotated as encoding expressed proteins with unknown function; one was annotated as encoding hypothetical protein, two were annotated as encoding transposon proteins with unknown function (Table 6). On the basis of cDNA sequences, qRT-PCR primers were designed for detecting the expression of the 12 genes in GH998 and $\mathrm{Y} 11$ under cold stress or different time periods (Fig. 5). Four genes were induced by cold stress in different degrees between Y11 and GH998. LOC_Os11g32940 had significantly higher expression levels at 1, 3, 6, 12, and $24 \mathrm{~h}$ and 2, 3, 4, 5, 6, and $7 \mathrm{~d}$ in $\mathrm{Y} 11$ than in GH998, and these levels encodes a OsFBX421-F-box domain containing protein. The expression of LOC_Os11g32880 in cold-tolerant Y11 was significantly higher than that in the cold-sensitive GH998 at 3 and 5 days, which encodes a DEAD-BOX ATP-dependent RNA helicase protein (Fig. 6b and Supplementary Fig. 4). These findings suggested that LOC_Os11g32940 and LOC_Os11g32880 may be candidate genes for QTL regulating CT in wild rice.

\section{Discussion}

Low-temperature stress during the germination stage in plants is frequent, especially at high altitudes in tropical and subtropical regions. Rice is sensitive to low temperature at germination, seedling, and booting stages, and cold stress leads to reduced yield (Yang et al. 2020; Borjas et al. 2016; Li et al. 2018; Zhang et al. 2017; Pan et al.2020). 


\section{High-density genetic mapping could be used to explore QTLs in wild rice}

Genome resequencing could effectively improve the QTL mapping efficiency. Hu et al. used whole genome resequencing to map a gene-regulated gummy stem blight in melon by using an ultra-dense genetic map consisting of 12,932 recombination bin markers (Zhongyuan Hu, 2017). Li et al. performed de novo assembly of a high-quality genome of SN265 and identified 79 QTLs related to 15 agronomic traits (Li et al. 2018). Li et al. integrated a molecular linkage map with 2972 bins; transcriptome and omic analyses were used to determine the major QTL RH8 for yield heterosis (Li et al. 2015). High-throughput sequencing was conducted for QTL of a number of large vascular bundle (LVB) analysis in a series of RILs, and the results showed that dep1 allele increased the number of LVBs (Cheng et al. 2019). The rapid development of sequencing technologies and publication of rice reference genome sequence made resulted in candidate gene identification, map-based gene cloning, and marker-assisted selection profitably (Li et al. 2018).

In the present study, whole genome resequencing (Bentley et al. 2006) was used to construct a highdensity bin-map and then identify two QTLs for CT at the germination stage in wild rice with a CSSL line. The germination rate of 27 lines under low temperature was higher than $90 \%$. Thus, whole genome resequencing associated with CSSLS could be fully excavated and used for wild rice gene resources.

\section{Mapping of cold-tolerant QTL at germination stages with different genetic backgrounds and research methods}

QTL mapping depended on marker density, the evaluation standard of population, and rice population. In previous studies, 200 traditional rice cultivars were evaluated using low-temperature germination, 1672 SNP markers were detected in QTL to be associated with LTG, and two wide regions of chromosomes 3 and 6 were consistently associated with rice LTG (Sales et al. 2017). Wang et al. used cold-tolerant phenotypes of temperate and tropical japonica rice cultivars and $44 \mathrm{~K}$ SNP chip dataset of rice diversity panel 1 to map 67 QTLs for CT at early seedling stages located on chromosome 11 (Wang et al. 2016). The germinating ability and seedling vigor under low temperature were measured using an RIL mapping population involving a US weedy rice accession "PSRR-1" and a rice cultivar "Bengal;" 49 QTL distributed over 10 chromosomes were identified for 11 traits (Borjas et al. 2016). Four QTLs with CT under low temperature at the germination stage were located on four chromosomes by NILs and linkage map (Satoh et al. 2016). Yang et al. constructed an RIL and a high-density genetic map to map six QTLs that explained $5.13-9.42 \%$ of the total PVE during the germination stage, the QTLs distributed on chromosome 9 (Yang et al. 2020). Several QTLs for CT under low temperature in rice near qLTG11 on chromosome 11 were reported in previous studies (Satoh et al. 2016; Jiang et al. 2017). Compared with the previously published QTL map of low-temperature germinability, the QTL in the present study was characterized in a narrow region by high-density linkage map from wild rice and cultivated rice. qLTG8 was in $195.5 \mathrm{~kb}$ with 32 genes on chromosome 8, while qLTG11 was mapped in $78.83 \mathrm{~kb}$ with 12 genes on chromosome 11. The alleles from Y11 of two QTL qLTG8 and qLTG11 could improve the seed 
germination rate under low temperature. This finding suggested that QTLs qLTG8 and qLTG11 and CSSLs could be highly valuable genetic factors for improving cold tolerance in rice breeding.

\section{Identified candidate genes of qLTG8 and qLTG11 could be useful for further research}

A search for candidate genes for $q L T G 8$ by using the Rice Genome Annotation Project database (http://rice.plantbiology.msu.edu/index.shtml) mapped 32 genes in the target region of the Nipponbare genome (Table 7). qRT-PCR was performed to investigate the expression of candidate genes under cold stress. The result showed that five genes were induced by cold stress at the germination stage. Furthermore, the nucleotide differences in the promoter region and the coding region of LOC_Os08g01120, LOC_Os08g01140, LOC_Os08g01390, LOC_Os08g01170, and LOC_Os08g01380 may be responsible for the different cold responses of Y11and GH998. LOC_Os08g01120 encodes a sulfate transporter, which is responsible for transporting sulfur in plants. Plants could convert inorganic sulfur into organic sulfur compounds via complex enzymatic steps (M. Aydın Akbudak, 2018 \#103). Sulfate transporter SULTR1;2 regulated the levels of glucosinolates in parts of the plant. LOC_Os08g01140 showed no annotation. LOC_Os08g01390 encoded a phosphatidylinositol-4-phosphate 5-kinase protein. In eukaryotic cells, phosphatidylinositol 4-phosphate 5-kinaseis a major enzyme that takes part in biosynthesizing the signaling molecule phosphatidylinositol 4,5-bisphosphate (Liu et al. 2016). The membrane phospholipid phosphatidylinositol 4,5-bisphosphate was required in cold- and mentholinduced activation of transient receptor potential melastatin 8 ion channel (Rohacs et al. 2020). LOC_Os08g01170 encoded an acetyltransferase. In Arabidopsis, CBF1 was regulated by cold-regulated genes depending on the activities of histone acetyltransferase (HAT) Gcn5 and transcriptional adaptor proteins Ada2 and Ada3 (Stockinger et al. 2001). Serotonin N-acetyltransferase is responsible for elevating chlorophyll synthesis during cold stress in rice (Kang et al. 2010). In cotton, HATs play vital roles in response to various stresses (salt, drought, cold, heavy metal, and DNA damage) and hormones, such as abscisic acid and auxin (Imran et al. 2019).

On the region of $q L T G 11, \angle O C \_O s 11 g 32940$ encodes an OsFBX421-F-box domain containing protein. In terms of F-box protein, Neilson et al. used cellulose synthase in linear models to predict cold-induced sweetening (Neilson et al. 2017). Saito et al. cloned and functionally validated the QTL Ctb1 encoding an F-box protein, which showed CT in the booting stage of rice (Saito et al. 2010). Venkatesh et al. identified that F-Box family genes, LTSF1 and LTSF2, regulated low-temperature stress tolerance in pepper (Capsicum chinense). They found that the pepper LTSF1 increased CT compared with non-transformed plants (Venkatesh et al. 2020). LOC_Os11g32880 encodes a DEAD-BOX ATP-dependent RNA helicase protein. AtRH7 is one of the Arabidopsis thaliana DEAD-box RNA helicases, an RNA chaperone involved in cold adaptation. Knockout AtRH7 mutant lines showed aberrant and lessened leaves with decreased size under cold stress. Taken together, the results suggested that AtRH7 affects rRNA biogenesis and plays an important role in plant growth under cold stress (Yuelin Liu, 2016). Wang reported that SHINY2 encodes a DEAD-(Asp-Glu-Ala-Asp) box RNA helicase that serves as a splicing factor required for proper splicing of cold-responsive genes (Wang et al. 2019). 
In summary, $q L T G 8$ and $q L T G 11$ were fine mapped and four candidate genes were identified. In future studies, functional analysis of $\mathrm{Y} 11$ and GH998 could be performed to validate the candidate genes via sequence analysis and genetic transformation.

\section{Materials And Methods Mapping Population}

In this study, the cold-tolerant wild rice Y11 (Oryza rufipogon Griff.) and the cold-sensitive rice variety GH998 were used as the donor and recipient, respectively, to develop the mapping population. Y11 crossed with $\mathrm{GH} 998$, and the progeny was continuously self-crossed to produce the $\mathrm{BC}_{3} \mathrm{~F}_{9}$ population. $\mathrm{A}$ set of CSSLs $\left(\mathrm{BC}_{3} \mathrm{~F}_{9}\right)$ consisting of 271 individuals was developed for subsequent analysis (Fig. 1). The population was developed in the experimental field (Nanning, $22.85^{\circ} \mathrm{N}, 108.26^{\circ} \mathrm{E}$ ) at the Rice Research Institute of Guangxi Academy of Agricultural Sciences in the summers of 2010-2019.

\section{Analysis of Whole Genome Resequencing Data}

Leaves of two parents and $\mathrm{BC}_{3} \mathrm{~F}_{9}$ individuals were collected the tillering stage and stored at $\mathrm{a}-80{ }^{\circ} \mathrm{C}$ freezer. The genomic DNA was prepared from each line of CSSL $\left(\mathrm{BC}_{3} \mathrm{~F}_{9}\right)$ via a modified CTAB method with small modification. Sequencing libraries were constructed using the Illumina HiSequation 2500 system. Raw reads from every sample were checked. Data were filtered to obtain high-quality reads. The clean reads were remapped on the reference genome used by BWA (Li et al. 2009). SNP was detected using GATK and Picard for base recalibration and variant calling and to strictly filter the SNPs (McKenna et al. 2010). The sequencing depth and genome coverage of each sample were statistically analyzed.

\section{Bin Map and Linkage Map Construction}

The markers were filtered using the following procedure to achieve map quality. First, the incongruent marker, which is parental homozygous, was selected. The parental marker depth should be no less than 10x. Removed non-chromosomal markers were present. Padding and calibration of SNP were described as a window of 15 SNPs and a step size of 1 SNP. The SNPs with segregation patterns aa $\times$ bb were used to construct the genetic map. Bin lengths of less than $10 \mathrm{~KB}$ were screened out. The, partial separation markers were used to filter with chi-square test. The linkage map contained 1484 bins, and an average of 123 bins was found on each chromosome. In accordance with the physical location of Bin marker on each chromosome, the genotype could visually represent the exchange and recombination of chromosomes during meiosis. Afterward, Bin was divided into 12 linkage groups. Finally, a genetic map was obtained by using the linear arrangement of markers within the linkage group and estimating the genetic distance between adjacent markers. The Bin number of each linkage group, the average length $(\mathrm{Mb})$ of Bin, the number of genes contained in Bin, the total plot distance, and the average plot distance were used for analysis. The monomer sources of each sample in the whole linkage group were counted to find the possible double exchange sites. The linkage of the map was assessed. The Spearman correlation 
coefficient of each linkage group and physical graph was counted, and linkage assessment was performed via collinear analysis of the location of the markers on the genome.

\section{Evaluation of CT in parents and population}

Mature seeds of 271 rice CSSLs were surface-sterilized with $10 \%$ solution of sodium hypochlorite for $15 \mathrm{~min}$ and washed three times with distilled water. The seeds of these 271 rice lines and parents were soaked at $25^{\circ} \mathrm{C}$ for $24 \mathrm{~h}$. All rice seeds were germinated in a greenhouse at a temperature of $28^{\circ} \mathrm{C} \pm 2{ }^{\circ} \mathrm{C}$ and relative humidity of $80-100 \%$. The seeds were prepared with a coleoptile length of $\geq 5 \mathrm{~mm}$. They were then treated under LT $\left(6^{\circ} \mathrm{C}\right)$ for 8 days and recovered for 7 days. Each replication consisted of 30 seeds in a glass dish $(9 \mathrm{~cm})$, as the experimental unit. Phenotypes were evaluated using the germination rate under low temperature. The collected samples were flash-frozen in liquid nitrogen and stored at $80^{\circ} \mathrm{C}$ until further use.

\section{qRT-PCR analysis of candidate genes}

Total RNA was extracted from the sprout by using the EasyPure Plant RNA Kit in accordance with the manufacturer's protocol, PrimeScript RT reagent Kit with gDNA Eraser was used in all RNAs for further analysis. The expression levels of candidate genes were determined via qRT-PCR with designed primers (Supplemental Table S1) and normalized to those of the housekeeping gene UBQ (Os03g0234200), which was used as the reference gene. RT-PCR was performed with a BioRad CFX96 Real-time System C1000 (Bio-Rad Laboratories, CA, USA) using Green qPCR MasterMix (MT521-01, Biomed, China) in accordance with the manufacturer's protocol. qRT-PCR was performed in $10 \mu \mathrm{L}$ mixtures: $5 \mu \mathrm{L}$ of $2 \times$ Green qPCR MasterMix, $1 \mu \mathrm{L}$ of cDNA, $0.25 \mu \mathrm{L}$ of each primer $(10 \mu \mathrm{M})$, and $3.5 \mu \mathrm{L}$ of $\mathrm{ddH}_{2} \mathrm{O}$. The amplification steps were $95^{\circ} \mathrm{C}$ for $30 \mathrm{~s}, 40$ cycles of $95^{\circ} \mathrm{C}$ for $5 \mathrm{~s}, 60^{\circ} \mathrm{C}$ for $30 \mathrm{~s}, 65^{\circ} \mathrm{C}$ for $5 \mathrm{~s}, 95^{\circ} \mathrm{C}$ for $15 \mathrm{~s}, 60^{\circ} \mathrm{C}$ for $30 \mathrm{~s}$, and $95^{\circ} \mathrm{C}$ for $15 \mathrm{~s}$. Each experiment was repeated three times, and qRT-PCR analysis was performed using $\Delta \Delta \mathrm{Ct}$ method.

\section{Abbreviations}

LT: Low-temperature; DSR: Direct-seeded rice; QTL: Quantitative trait loci; CSSL: Chromosome segment substitution line; SNP: Single nucleotide polymorphism; PVE: Phenotypic variation explained; LVG: Lowtemperature vigor of germination; CIVG: Cold response index for vigor of germination; GWAS: Genomewide association studies; SSSLs: Single segment substitution lines; LTG: Low-temperature germinability; RILs: Recombinant inbred lines; CT: Cold tolerance; qRT-PCR: Quantitative real-time PCR; LVBs: Large vascular bundles; LTG: Low-temperature germination.

\section{Declarations}

\section{Acknowledgments}

We thank Dr Zichao Li (China Agricultural University, China) for the constructive suggestions. 


\section{Author Contribution Statements}

G-F D, D T L and G-X D designed and supervised the research. L-J G and C L performed the experiments. B-X N, C-C H analyzed data. X-Z X, Z-Q Z constructed the population. Y-H P and X-H Y wrote the paper. All authors have read and approved the manuscript.

\section{Funding}

The present study was supported by the Key Project of Guangxi Natural Science Foundation (Grant No. 2018GXNSFDA281053, 2019GXNSFBA245006), the Project of the central government guides the development of local science and technology (GuikeZY20198015), the Open Project of Guangxi Key Laboratory of Rice Genetics and Breeding and Guangxi Major Scientific and Technological Innovation base (Grant No. 2018-05-06-KF08), National Natural Science Foundation of China (Grant Nos. NSFC32060452, NSFC32060454, NSFC32060476), the Base Business Project of Guangxi Academy of Agricultural Sciences (Grant No. GuiNongKe 2020YM72, GuiNongKe 2019M07) and The funding bodies had no role in the design of the study; collection, analysis, and interpretation of data; and in writing the manuscript.

\section{Ethics Approval and Consent to Participate}

Not applicable.

\section{Consent for Publication}

Not applicable.

\section{Competing Interests}

The authors declare that they have no competing interests

\section{References}

McKenna A, Hanna M, Banks E, Sivachenko A, Cibulskis K, Kernytsky A, Garimella K, Altshuler D, Gabriel S, Daly M and DePristo MA (2010). The Genome Analysis Toolkit: a MapReduce framework for analyzing next generation DNA sequencing data. Genome Res 20:1297-1303

Liu AZ, Sui DX, Wu DQ and Hu J (2016). The activation loop of PIP5K functions as a membrane sensor essential for lipid substrate processing. Science advances 2(11): e1600925

Borjas AH, Leon TBD \&Subudhi PK (2016) Genetic analysis of germinating ability and seedling vigor under cold stress in US weedy rice. Euphytica 208:251-264.

Wang BS, Chai HX, Zhong YL, Shen Y, Yang WN, Chen JP, Xin ZG, Shi HZ (2019) The DEAD-box RNA helicase SHI2 functions in repression of salt-inducible genes and regulation of cold-inducible gene 
splicing. Journal of Experimental Botany 71(4): 1598-1613.

Bentley DR (2006) Whole-genome re-sequencing. Current Opinion in Genetics \& Development 16(6):545552

Li BB, Lu XQ, Dou JL, Aslam A, Gao L, Zhao SJ, He N and Wenge Liu WG (2018) Construction of A HighDensity Genetic Map and Mapping of Fruit Traits in Watermelon (Citrullus Lanatus L.) Based on WholeGenome Resequencing. International Journal of Molecular Sciences 19(10): 3268

Fei C, Geng X, Xu ZJ \& Xu Q (2019). Multiple areas investigation reveals the genes related to vascular bundles in rice. Rice 12, 17.

Liu CT, Schläppi MR, Mao BG, Wang W, Wang AJ, Chu CC (2019) The bZIP73 transcription factor controls rice cold tolerance at the reproductive stage. Plant Biotechnology Journal 17, 1834-1849.

Wang D, Liu JL, Li CG, Kang HX, Wang Y, Tan XQ, Liu MH, Deng YF, Wang ZL, Liu Y, Zhang DY, Xiao YH \& Wang GL (2016) Genome-wide association mapping of cold tolerance genes at the seedling stage in rice. Rice 9, 61

Li DY, Huang ZY, Song SH, Xin YY, Mao DH, Lv QM, Zhou M, Tian SM, Tang MF, Wu Q, Liu X, Chen TT, Song XW, Fu XQ, Zhao BR, Liang CZ, Li AH, Liu GZ, Li SG, Hu SN, Cao XF, Yu J, Yuan LP, Chen CY, and Zhu LH (2015) Integrated analysis of phenome, genome, and transcriptome of hybrid rice uncovered multiple heterosis-related loci for yield increase. PNAS 113 (41): E6026-E6035.

Mao DH, Xin YY, Tan YJ, Hu XJ, Bai JJ, Liu ZY, Yu YL, Li LY, Peng C, Fan T, Zhu YX, Guo YL, Wang SH, Lu DP, Xing YZ, Yuan LP, and Chen CY (2019) Natural variation in the HAN1 gene confers chilling tolerance in rice and allowed adaptation to a temperate climate. PNAS 116 (9): 3494-3501

Stockinger EJ, Mao YP, Regier MK, Triezenberg SJ, Thomashow MF (2001) Transcriptional adaptor and histone acetyltransferase proteins in Arabidopsis and their interactions with CBF1, a transcriptional activator involved in cold-regulated gene expression. Nucleic Acids Research 29(7):1524-1533.

Sales E, Viruel J, Domingo C, Marqués L (2017). Genome wide association analysis of cold tolerance at germination in temperate japonica rice (Oryza sativa L.) varieties. PLoS One 12, e0183416.

Lu GW, Wu FQ, Wu WX, Wang HJ, Zheng XM, Zhang YH, Chen XL, Zhou KN, Jin MN, Cheng ZJ, Li XY, Jiang L, Wang HY, Wan JM (2014). Rice $L T G 1$ is involved in adaptive growth and fitness under low ambient temperature. The Plant Journal 78, 78(73): 468-480.

Han LZ, Zhang YY, Qiao LY, Cao GL, Zhang SY, Kim JH, Koh HJ (2006). Genetic and QTL Analysis for LowTemperature Vigor of Germination in Rice. Acta Genetica Sinica 33(11):998-1006.

Venkatesh J, Kang MY, Liu L, Kwon JK and Kang BC (2020). F-Box Family Genes, LTSF1 and LTSF2, Regulate Low-Temperature Stress Tolerance in Pepper (Capsicum chinense). Plants 9(9):1186 
Li JL, Pan YH, Guo HF, Zhou L, Yang SM, Zhang ZY, Yang JZ, Zhang HL, Li JJ, Zeng YW\& Li ZC(2018). Fine mapping of QTL qCTB10-2 that confers cold tolerance at the booting stage in rice. Theoretical and Applied Genetics 131, 157-166

Yang J, Li D, Liu H, Liu YZ, Huang M, Hui Wang, Chen ZQ, Guo T (2020). Identification of QTLs involved in cold tolerance during the germination and bud stages of rice (Oryza sativa L.) via a high-density genetic map. Breeding Science 709(3): 292-302.

Neilson J, Lagüe M, Thomson S, Aurousseau F, Murphy AM, Bizimungu B, Deveaux V, Bègue Y, Jacobs JME \& Tai HH (2017). Gene expression profiles predictive of cold-induced sweetening in potato. Functional \& Integrative Genomics 17, 459-476

Junliang Zhao, Zhang SH, Dong JF, Yang TF, Mao XX, Liu Q, Wang XF and Liu B (2017). A novel functional gene associated with cold tolerance at the seedling stage in rice. Plant Biotechnology Journal $15,1141-1148$.

Saito K, Hayano-Saito Y, Kuroki M, and Sato Y (2010) Map-based cloning of the rice cold tolerance gene Ctb1. Plant Science 179(1): 97-102

Fujino K, Sekiguchi H, Matsuda Y, Sugimoto K, Ono K, and Yano M (2008) Molecular identification of a major quantitative trait locus, $q L T G 3-1$, controlling low-temperature germinability in rice. PNAS 105 (34):12623-12628.

Kang K, Lee K, Park S, Kim YS, Back K (2010) Enhanced production of melatonin by ectopic overexpression of human serotonin $\mathrm{N}$-acetyltransferase plays a role in cold resistance in transgenic rice seedlings. Journal of Pineal Research 49, 176-182.

Yang LM, Liu HL, Lei L, Zhao HW, Wang JG, Li N, Sun J, Zheng HL, Zou DT (2018) Identification of QTLs controlling low-temperature germinability and cold tolerance at the seedling stage in rice (Oryza Sativa L.). Euphytica 214, 13.

Li LY, Chen HP, Mao DH (2019) Pyramiding of rapid germination loci from Oryza Sativa cultivar 'Xieqingzao $\mathrm{B}^{\mathrm{\prime}}$ and cold tolerance loci from Dongxiang wild rice to increase climate resilience of cultivated rice. Mol Breeding 39, 85

Li H, Durbin R (2009) Fast and accurate short read alignment with Burrows Wheeler Transform. Bioinformatics 25(14):1754-1760

Imran Muhammad, Sarfraz S, Farooq MA, Naeem MK, Widemann E, Bakhsh A, Jensen KB and Wang RC (2019) Comparative Genome-wide Analysis and Expression Profiling of Histone Acetyltransferase (HAT) Gene Family in Response to Hormonal Applications, Metal and Abiotic Stresses in Cotton. International Journal of Molecular Sciences 20(21): 5311 
Xiao N, Gao Y, Qian HJ, Gao Q, Wu YY, Zhang DP, Zhang XX, Yu L, Li YH, Pan CH, Liu GQ, Zhou CH, Jiang M, Huang NS, Dai ZY, Liang CZ, Chen Z, Chen JM, Li AH (2018) Identification of genes related to cold tolerance and a functional allele that confers cold tolerance. Plant Physiology 177, 1108-1123

Jiang NF, Shi SL, Shi H, Khanzada H, Wassan GM, Zhu CL, Peng XS, Yu QY, Chen XR, He XP, Fu JR, Hu LF, Xu J, Ouyang LJ, Sun XT, Zhou DH, He HH and Bian JM (2017). Mapping QTL for seed germinability under low temperature using a new high-density genetic map of rice. Frontiers in Plant Science 8, 1223.

Pan Yh, Zhang HL, Zhang DL, Li JJ, Xiong HY, Yu JP, Li JL, Rashid MAR, Li GL, Ma XD, Cao GL, Han LZ, Li ZC (2015) Genetic analysis of cold tolerance at the germination and booting stages in rice by association mapping. PLoS One 10, e0120590

Cruz RPD, Sperotto RA, Cargnelutti D, Adamski JM, Freitasterra TD, Fett JP (2013) Avoiding damage and achieving cold tolerance in rice plants 2, 96-119.

Liu LY, Rohacs T (2020). Regulation of the cold-sensing TRPM8 channels by phosphoinositides and Gqcoupled receptors. Channels 14(1):79-86

Yang TF, Zhang SH, Zhao JL, Liu Q, Huang ZH, Mao XX, Dong JF, Wang XF, Zhang GQ, Liu B (2016) Identification and pyramiding of QTLs for cold tolerance at the bud bursting and the seedling stages by use of single segment substitution lines in rice (Oryza sativa L.). Mol Breeding 36:96

Satoh T, Kouichi T, Kawamoto T, Matsumoto S, Satoh-Nagasawa N, Ueda K, Sakurai K, Watanabe A, Takahashi H, Akagi H (2016) Identification of QTLs controlling low-temperature germination of the East European rice (Oryza sativa L.) variety Maratteli. Euphytica 207, 245-254

Li XK, Wu L, Wang JH, Sun J, Xia XH, Geng X, Wang XH, Xu ZJ, and Quan Xu Q (2018) Genome sequencing of rice subspecies and genetic analysis of recombinant lines reveals regional yield- and quality-associated loci. BMC Biology 16, 102

Xu M, Ye XF, Wang WY, Wei CC, Zhang JW, and Tu JM (2015) Genetic analysis and molecular mapping of a high threshold and low temperature-sensitive mutant in rice (Oryza sativa L.) at the seedling stage. Euphytica 203, 71-82

Ding YL, Shi YT, Yang SH (2019) Advances and challenges in uncovering cold tolerance regulatory mechanisms in plants. New Phytologist 222, 1690-1704

Pan YH, Liang HF, Gao LJ, Dai GX, Chen WW, Yang XH, Qing DJ, Gao J, Wu H, Huang J, Zhou WY, Huang CC, Liang YT \& Deng GF (2020) Transcriptomic profiling of germinating seeds under cold stress and characterization of the cold-tolerant gene LTG5 in rice. BMC Plant Biology 20, 371.

Liu YL, Tabata D, Imai R (2016). A cold-inducible DEAD-Box RNA helicase from arabidopsis thaliana regulates plant growth and development under low temperature. PLoS One 11, e0154040 
Ma Yun, Dai X, Xu YY, Luo W, Zheng XM, Zeng DL, Pan YJ, Lin XL, Liu HH, Zhang DJ, Xiao J, Guo XY, Shujuan Xu, Niu YD, Jin JB, Zhang H, Xu X, Li LG, Wang W, Qian Q, Ge S, Chong K (2015). COLD1 Confers Chilling Tolerance in Rice. Cell 160(6):1209-1221

Zhang ZY, Li JJ, Pan YH, Li JL, Zhou L, Shi HL, Zeng YW, Guo HF, Yang SM, Zheng WW, Yu JP, Sun XM, Li GL, Ding YL, Ma L, Shen SQ, Dai LY, Zhang HL, Yang SH, Guo Y \& Li ZC (2017) Natural variation in CTB4a enhances rice adaptation to cold habitats. Nat Commun 8, 14788

Hu ZY, Deng GC, Mou HP, Xu YH, Chen L, Yang JH, and Zhang MF (2017) A re-sequencing-based ultradense genetic map reveals a gummy stem blight resistance-associated gene in Cucumis melo. DNA Research 25(1): 1-10

\section{Tables}

Due to technical limitations, the Tables are only available as a download in the supplemental files section

\section{Figures}




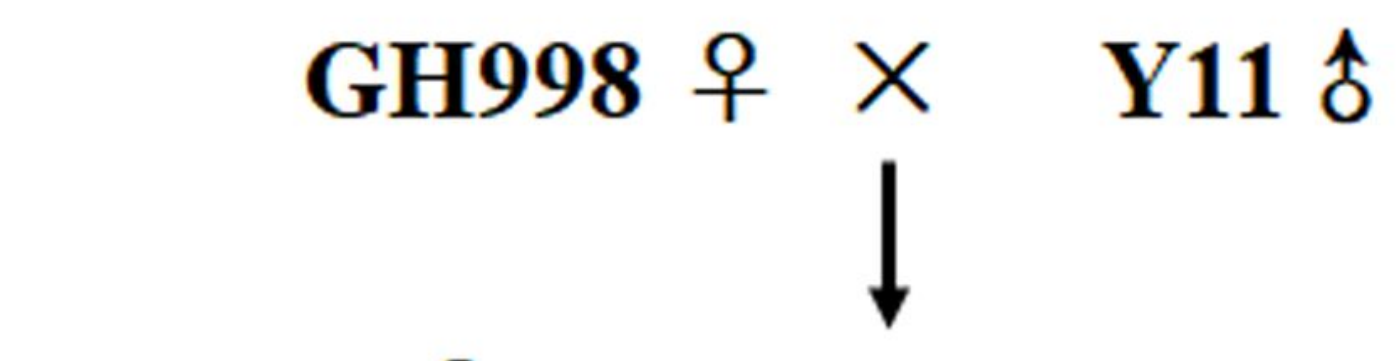

\section{GH998 우 $\times \quad \mathrm{BC}_{1} \mathrm{~F}_{1}$ \\ Backcross}

$\mathrm{BC}_{3} \mathrm{~F}_{1}$

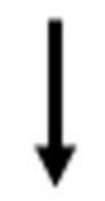

$\mathrm{BC}_{3} \mathrm{~F}_{9}$ (Re-sequencing)

$\downarrow$

$\mathrm{BC}_{3} \mathrm{~F}_{11}$ (QTL-mapping)

Figure 1

Construction of the CSSLs. 


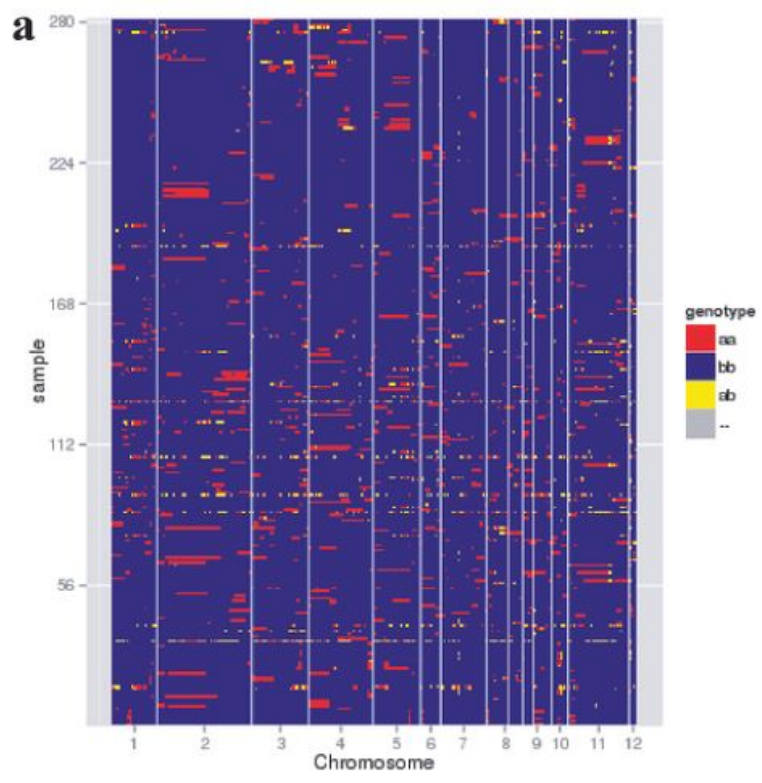

b

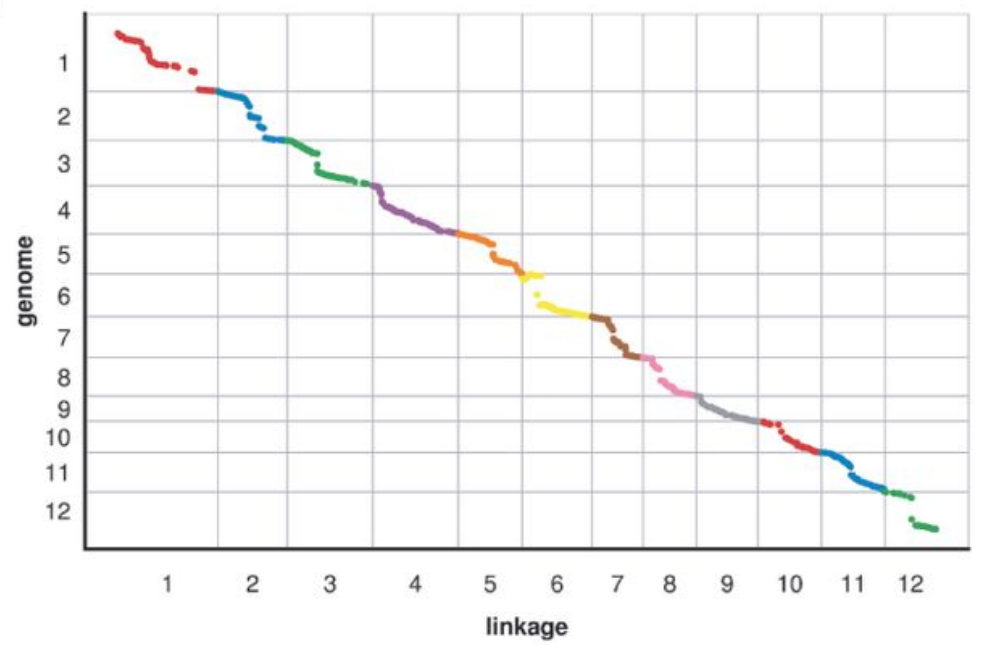

c

Genetic Map

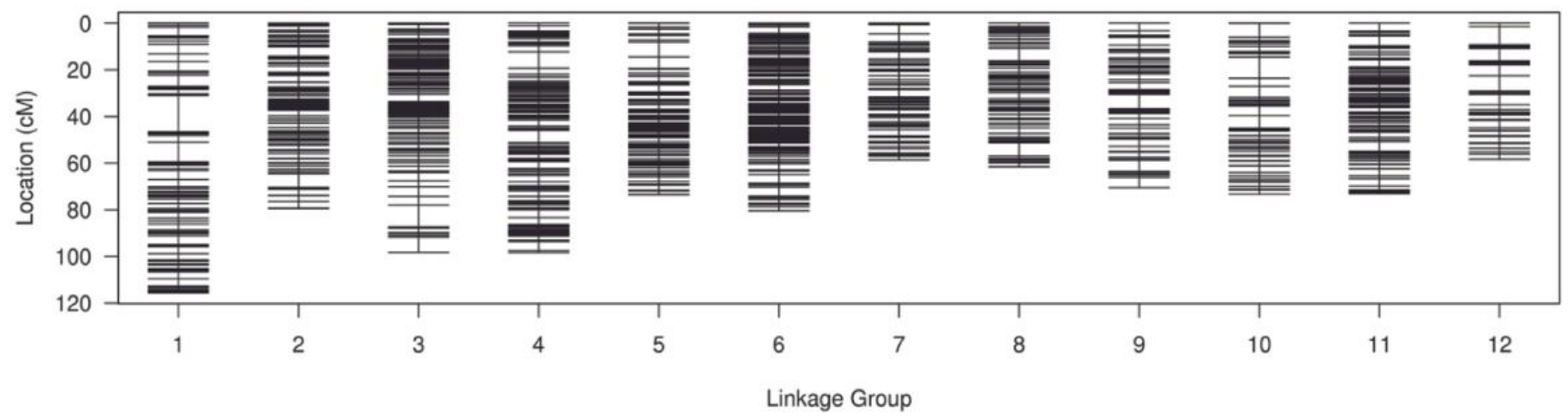

Figure 2

Construction of graphical genotype and linkage group. a: Construction of graphical genotype. Note: Red means the chromosome come from Y11, bule means the chromosome come from GH998, orange means the chromosome is heterozygous. Abscissa is chromosome, ordinate is CSSLs. b: Genetic map and genomic collinearity map. Note: Abscissa is genetic distance of each linkage group, ordinate is physical length of linkage group. The drop is the spearman coefficient. c: High density genetic map.

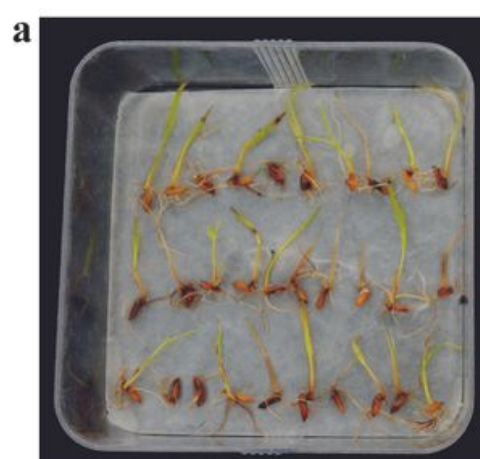

GH998

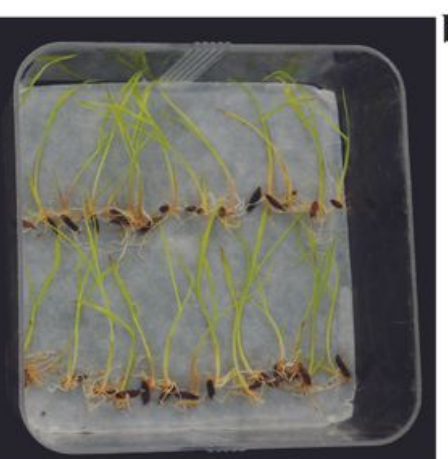

Y11 
Figure 3

Germination rate under low temperature of parents and CSSLs. a: Germination rate under low temperature of GH998 and $\mathrm{Y} 11$ at germination stage. b: Statistical comparison of the mean germination rate between GU998 and Y11. c: Frequency distribution of mean germination rate under low temperature of CSSLs population.

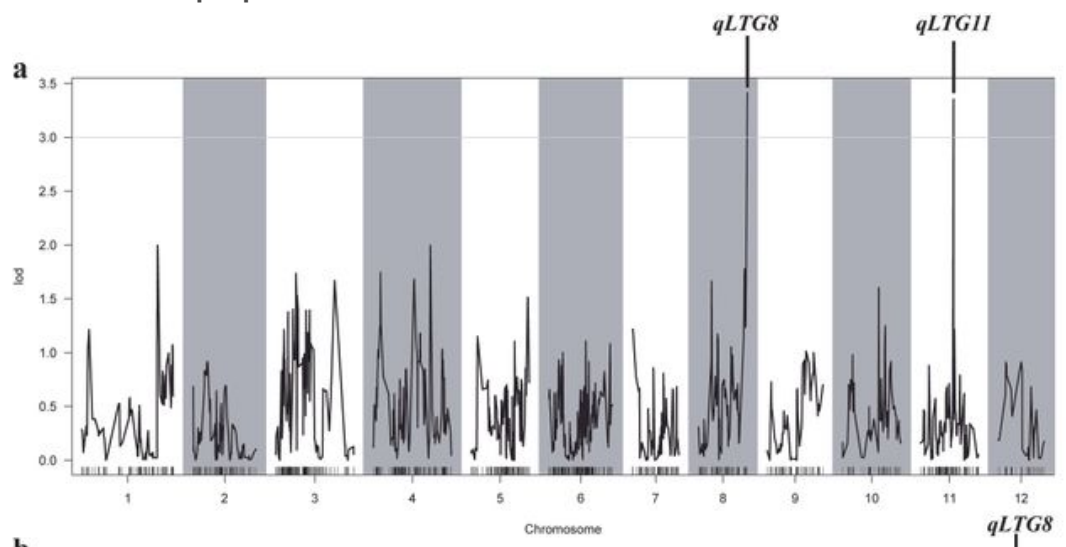

b
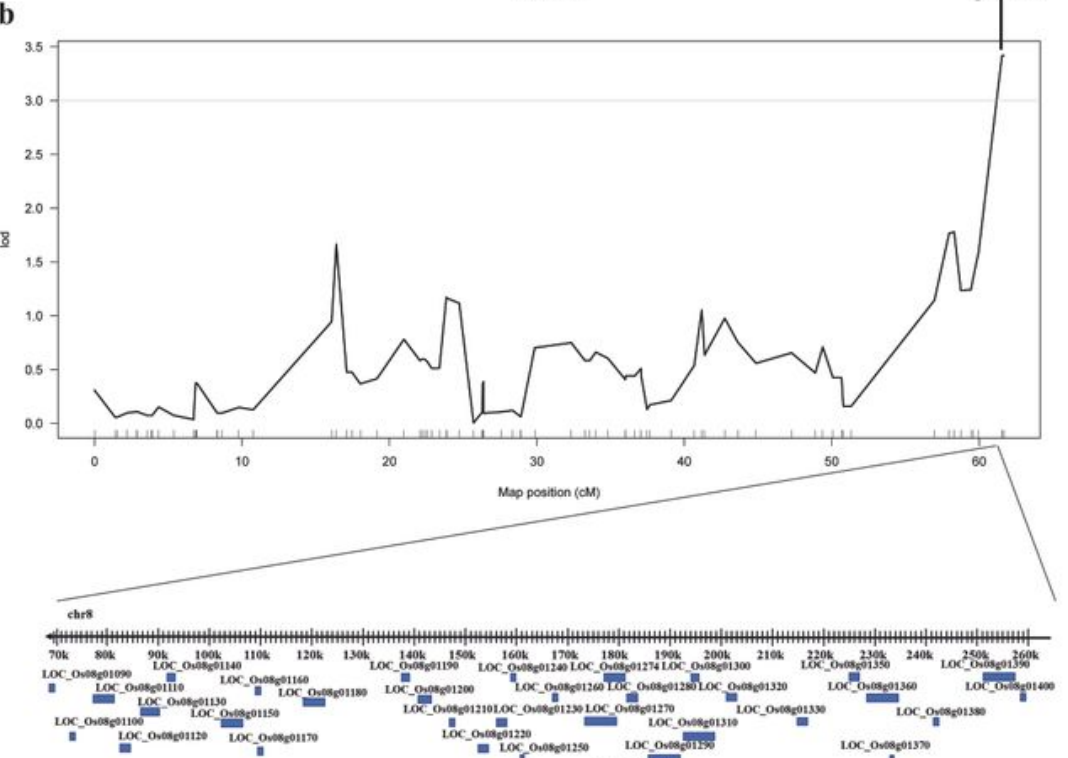

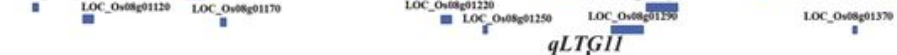

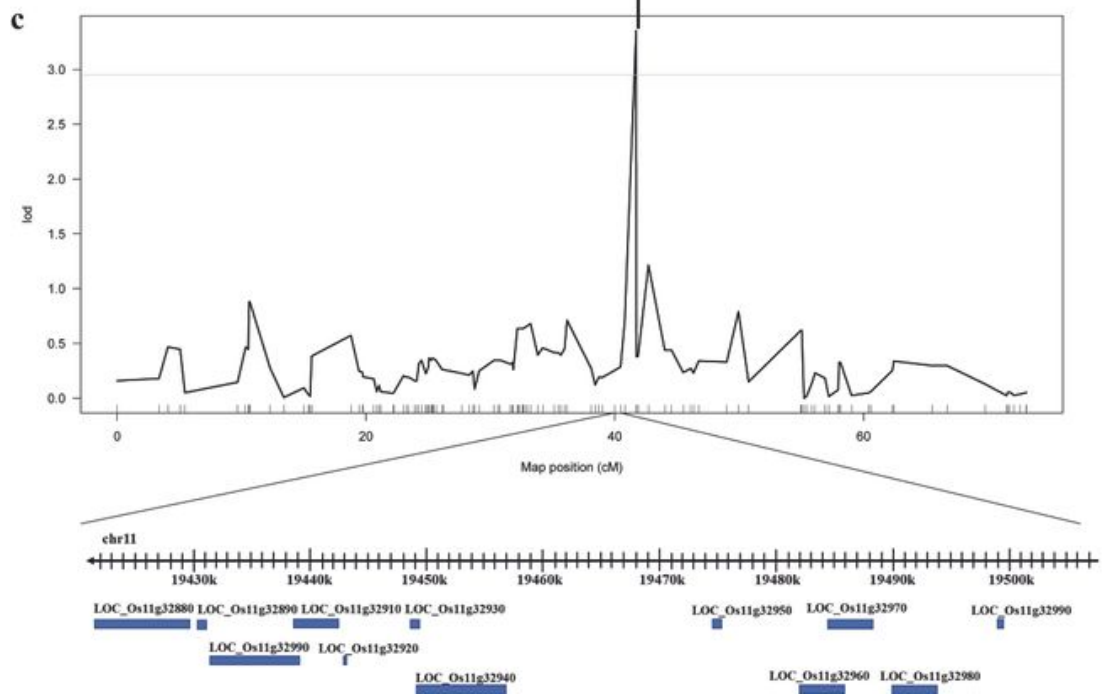

Figure 4 
Fine mapping of qLTG8 and qLTG11 using CSSLs and re-seq. a: A linkage map construction of qLTG8 and qLTG11 was developed for chromosome 1-chromosome 12. b: The qLTG8 a locus was narrowed to a $195.8 \mathrm{~kb}$ distance and contain 32 candidate genes. c: The qLTG11 a locus was narrowed to a $78.83 \mathrm{~kb}$ distance and contain 12 candidate genes.

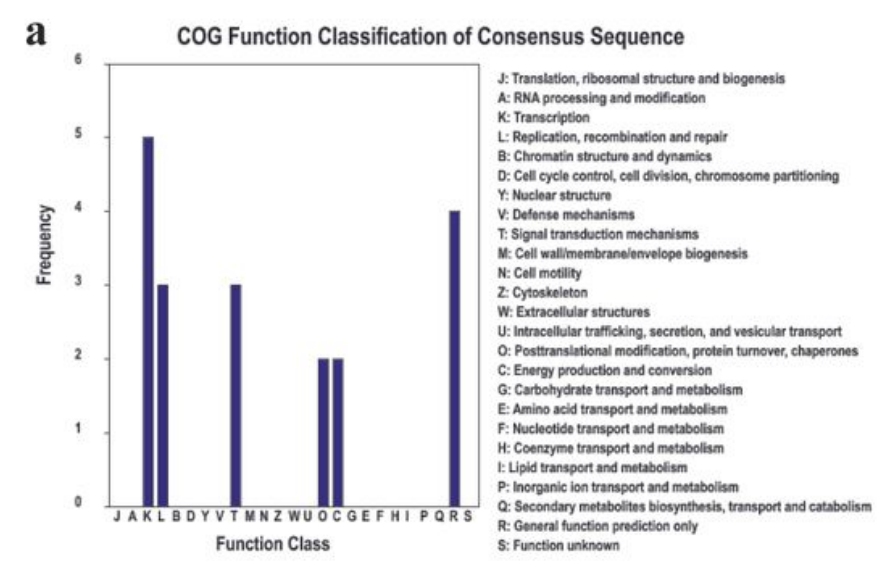

$$
\text { c }
$$

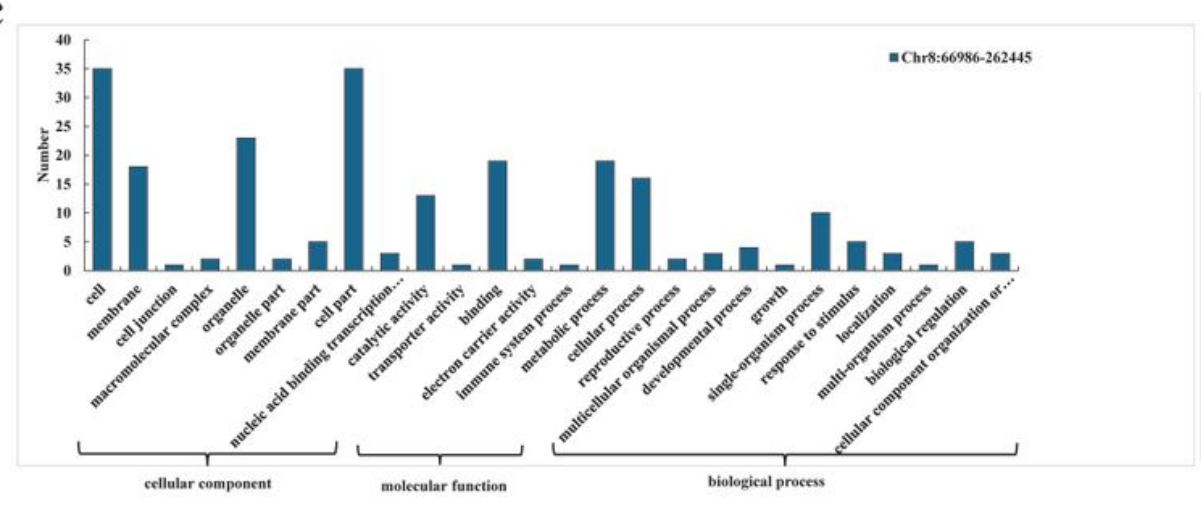

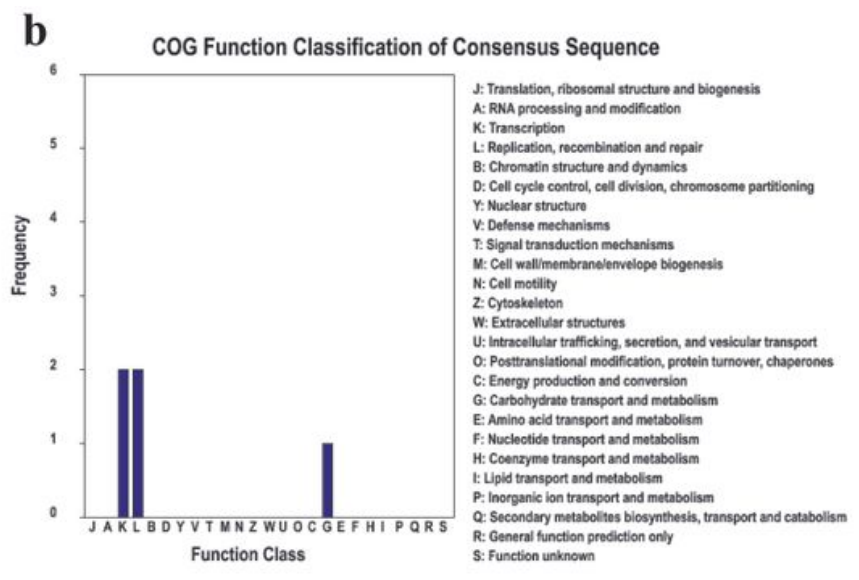

d

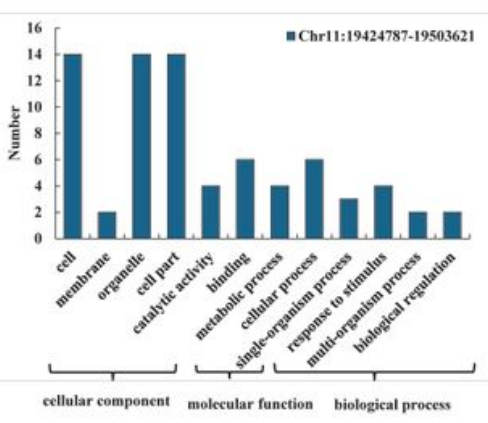

d

Figure 5

Candidate gene analysis. a: COG function classification of consensus sequence in qLTG8. b: COG function classification of consensus sequence in qLTG11. c: The number and gene function of Candidate gene in qLTG8.d: The number and gene function of Candidate gene in qLTG11. 

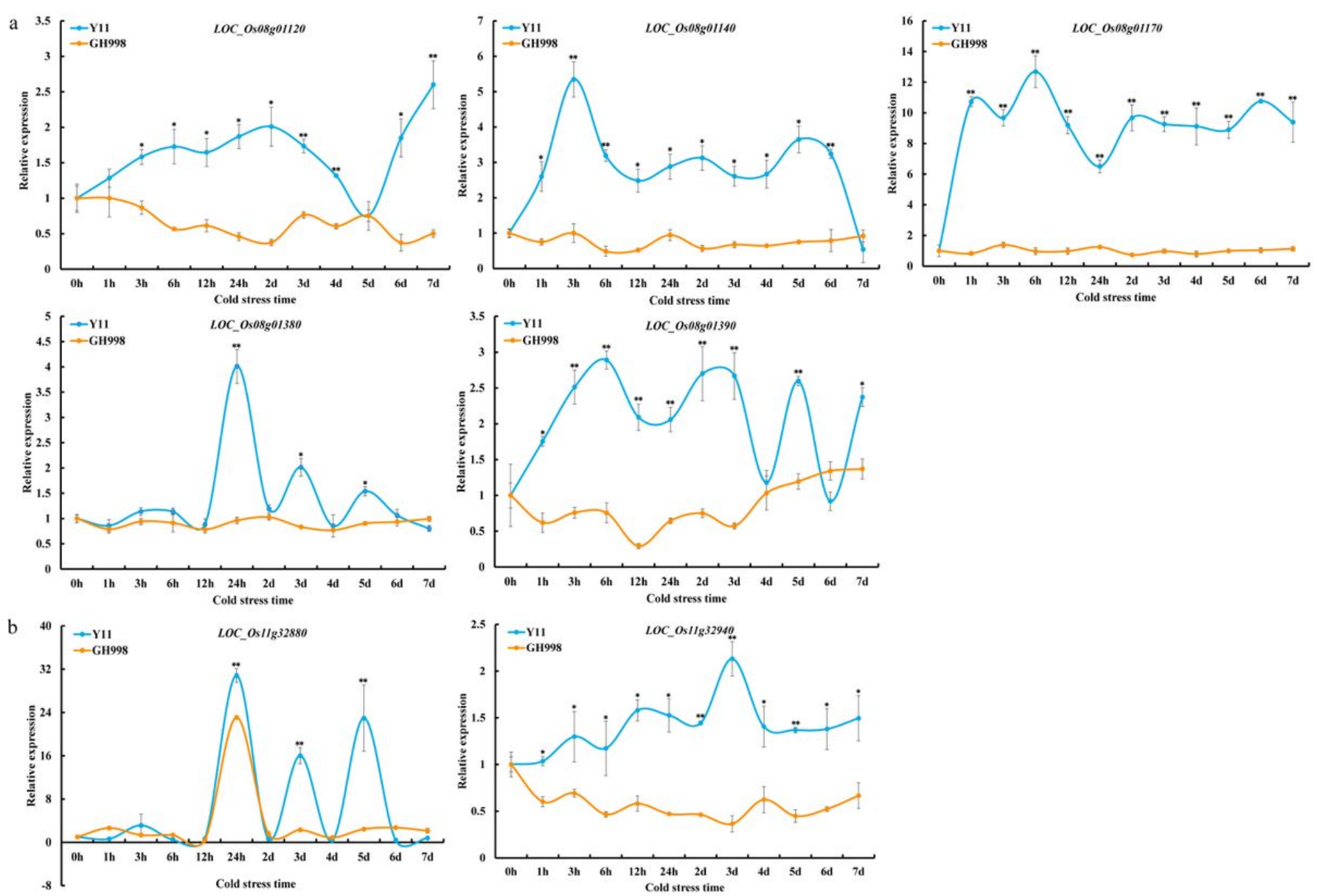

Figure 6

Expression analysis of candidate genes for $\mathrm{GH} 998$ and $\mathrm{Y} 11$ subjected to a $\mathrm{LT}\left(6^{\circ} \mathrm{C}\right)$ for different time periods by qRT-PCR. a: Expression analysis of candidate genes for GH998 and Y11 of qLTG8.b:

Expression analysis of candidate genes for GH998 and Y11 of qLTG11. Data represent mean \pm SD $(n=$ $3),{ }^{\star *} \mathrm{P}<0.01,{ }^{*} \mathrm{P}<0.05$, Student's $\mathrm{t}$ test.

\section{Supplementary Files}

This is a list of supplementary files associated with this preprint. Click to download.

- Table1.xlsx

- Table2.xlsx

- Table3.xlsx

- Table4.xlsx

- Table5.xlsx

- SupplementalTableS1.xlsx 
- SupplementaryFig1.jpg

- SupplementaryFig2.jpg

- SupplementaryFig3.jpg

- SupplementaryFig4.jpg 